\title{
Environmental Regulation: Choice of Instruments under Imperfect Compliance*
}

\author{
Inés Macho-Stadler ${ }^{\dagger}$ \\ Running Title: Environmental Regulation
}

March 11, 2006

*This paper originated from the AEE/REE presidential address for the XXX Simposio de Análisis Económico (Murcia, 2005). It builds on the research project on optimal enforcement in environmental problems done in collaboration with David Pérez-Castrillo. I would like to thank David Pérez-Castrillo and Pau Olivella for their useful comments. I gratefully acknowledge the financial support from the Ministerio de Ciencia y Tecnología (BEC2003-01132) and the Generalitat de Catalunya (Barcelona Economics - CREA and 2005SGR-00836).

†Dept. of Economics \& CODE, Universitat Autònoma de Barcelona, 08193 Bellaterra (Barcelona), Spain. Email: ines.macho@uab.es. Telephone: 349358118 12. Fax: 34935812461. 


\begin{abstract}
Compliance is an important issue in environmental regulation. We discuss some of the key elements of the problem and analyze a situation where emissions are not random and firms are risk-neutral. We study the firm's decision on emissions and compliance when the environmental regulation is based on standards and the enforcement agency audits the firm with a certain probability. We compare total emissions when environmental regulation is based on different instruments: standards, taxes, and tradable permits. We show that when compliance is an issue, environmental taxes are superior to the other instruments, and we analyze the (static) efficiency of the solution.
\end{abstract}

JEL Classification numbers: K32, K42, D82.

Keywords: environmental regulation, audits and compliance, environmental instruments. 


\section{Introduction}

It has long been recognized that enforcement is an important element of regulatory policy design. In the environmental economics literature the traditional approach used to discuss the optimal environmental policy has been to assume that polluters comply with the environmental regulations. However, full compliance can only be achieved under perfect and costless monitoring and with penalties dissuasive enough to induce agents to respect the rules.

In recent years there has been rapid growth in both theoretical and empirical studies of monitoring and enforcement. ${ }^{1}$ This is related to both the growth of the law and economics literature, and to the increased interest in the issue of law enforcement, as well as to the fact that the impact of enforcement agencies and regulatory concerns has increased. In addition, ignoring the possibility that firms adopt opportunistic behaviors leads to results that are far from desirable. If environmental regulation is stringent but there is no enforcement mechanism ensuring that firms comply, the results will be completely different from the aims. Monitoring and enforcement concerns should influence agencies' choice of regulatory instruments and in some instances, even the decision of whether to regulate at all.

Since Becker's (1968) analysis of crime, the combination of probability of detection and fines has been the basis for deterrence. Following this, the mechanism agencies use to compel firms to comply should combine monitoring (such as inspections) and enforcement (such as sanctions or remedial actions). The literature that follows this view consistently concludes that increasing the monitoring pressure or the penalty helps to increase compliance.

Studying how the different regulatory instruments work under imperfect compliance is important in determining which to choose. To perform this study one needs to describe (i) the type of pollution to analyze; (ii) the instrument or instruments to be considered; (iii) the behavior of firms that pollute; and (iv) the motivation of the enforcement agency.

\footnotetext{
${ }^{1}$ The compliance issue based on monitoring (or inspections) and fines is of general interest in many fields. For a general review of the compliance literature, see Polinsky and Shavell (2000). For environmental problems, Cropper and Oates (1992), Cohen (1999), Heyes (2000), and Sandmo (2000) provide extensive reviews of the literature.
} 
The remainder of this Section includes a simple typology for the first two aspects.

\subsection{Types of pollution}

Emissions can be classified according to different criteria. With respect to our main question, enforceability, a key criterion is how effective monitoring is when applied to the firm. For this dimension, one important aspect concerns how long the pollutant lasts in a particular place. Emissions can be classified as fugitive (like sulfur dioxide or $\mathrm{SO}_{2}$ ), or persistent (like an oil spill). Another important criterion concerns the difficulty in determining the agent responsible for the emissions: some emissions are observable to the auditor (point-source pollution discharges), while others are non-observable to the auditor (non point sources, such as pesticides). Finally, concerning the possibility of manipulating the emissions, one can speak of concealable emissions (dumping the residuals into the sea at night) and non concealable ones (gasses generated in a coal-fired utility boiler). Which aspect an agency concentrates on depends on the type of emissions considered. For some emissions, monitoring can be effective while for others the regulator needs to take into account the limits of enforceability.

Another important aspect the analysis must take into account is how much control do firms have on the pollution. Some emissions come from the intentional decision to violate the law (by not complying with the regulatory standard or by not paying the appropriate emission's fee), while others are of stochastic nature (in such a way that it is not easy to know whether the existence of pollution is due to a willful violation of the law, some negligent behavior, or a random act of nature).

Finally, one has to know who is involved in the activity. Some environmental problems come from few and big agents (electricity firms), while in some other cases the emissions come from many small agents (car emissions).

The previous characteristics influence the effectiveness of the monitoring strategy, the responsibilities of the firms, and in turn the type of policy instruments that are more useful in each case. Intuitively, a pollutant that is very difficult to observe should be controlled indirectly, via taxes on the input (e.g., fertilizers), or on the output of the firm (e.g., pesticides). These reasons may also explain why, for persistent emissions, the regulator 
may find self-reporting by firms useful. It can also explain why for some emissions where the identity of the responsible firm is difficult to observe or for emissions easy to conceal, the deposit-refund system may be useful (glass bottles, car batteries, motor oil), in the same way that for some big and few agents, installing particular technologies may be a good instrument. Finally, for other problems, the regulator may try to build a culture of compliance via ecological or green labeling to affect consumers' behavior or investor' decisions.

For all of these cases monitoring is still a concern. Hence, the study of the imperfect compliance case will allow better design of environmental policies.

\subsection{Environmental policy instruments}

Environmental policy instruments may take different forms:

(a) Command-and-control instruments, that include prohibitions (of inputs, processes or products), technology specification (for production, recycling, or waste treatment), and performance specification (for emission standards, or discharge quotas).

(b) Market-based mechanisms, that include tradable performance specification (tradable permits, catch quotas, or water-shares), pollution taxes, emission taxes, effluent taxes, input taxes, pollution charges (access fees or users charges), subsidies on abatement of emissions (tax deductions, credits, or soft loans).

(c) Other mechanisms, such as property rights (ownership or use rights), provision of information (to polluters, investors, or consumers) or deposit-refund systems (beer and soft drink bottles, old cars, car batteries, etc).

Environmental instruments are also often divided into quantity instruments (standards, or permits) and price instruments (taxes). Under full compliance, economists often argue about the advantage of market-based over command-and-control instruments because of their "anonymity": these instruments are set without specifying a particular rule for each agent. However, it is well-known that under certainty and full information environmental one has that taxes, standards and tradable permits lead to similar results. In addition, market-based instruments (taxes and tradable permits) lead to equalization of marginal abatement costs across firms, which allows them to reach the aggregate emis- 
sion target at the lowest costs. This property, referred to as (static) efficiency, can also be reached via standards, but then the emissions of each firm have to be set in order to satisfy the efficiency constraint. ${ }^{2}$

There is a growing literature on environmental regulations and more recently on the enforcement issue. ${ }^{3}$ However, the analysis of which instrument is more effective under imperfect compliance has been the subject of only a few papers. ${ }^{4}$

This paper's purpose is not to provide another review of the literature but, in contrast, to present a model in which the enforcement issue is analyzed and to explain how this type of analysis can help in comparing the performance of different environmental instruments. With this objective, in what follows, I will concentrate on non random pollution, where monitoring is costly but allows agencies to determine the firms' emissions. Section 2 presents the model and the behavior of the firms under emission standards. Section 3 compares total emissions when the regulator uses standards, taxes, or tradable permits as an instrument, and also discusses the efficiency distortion induced by compliance problems. Section 4 presents some conclusions and some open questions for future research.

\footnotetext{
${ }^{2}$ In other words, using non-uniform standards might reach the same solution but would require much more information about firms. Under uncertainty about marginal abatement benefits and full compliance, on the one hand, permits and standards (quantity instruments) guarantee emission levels but induce uncertain marginal abatement benefits. On the other hand, taxes (price instruments) place an upper bound on marginal abatement benefits but induce an uncertain effect on the total pollution level. Which instrument is more appropriate depends on the relative slope of the marginal abatement benefits for firms and the marginal damage costs for society (see Weitzman, 1974)

${ }^{3}$ There are also significant empirical contributions. Some empirical papers (see, for example, Dasgupta et al., 2001, and Foulon et al., 2002) document the effect of monitoring and enforcement actions on the level of pollution emissions (for a review, see Cohen, 2000). They provide evidence that both inspection and the threat of an inspection are useful in reducing pollution emissions.

${ }^{4}$ Some papers compare the different instruments under imperfect compliance, such as Sandmo (2002) and Montero (2002). They discuss different instruments and show which one, combined with an audit strategy, provides a better outcome if firms intentionally violate the law by not complying with a regulatory standard or by not paying the appropriate emission taxes. Il present their results at the end of Section 3.
} 


\section{The Model and the Firm's decision}

This section presents the basic model and considers the decision process for a single competitive firm. The firm chooses the level of emission $e$, where $e \in \mathbb{R}^{+}$. The firm's benefits from emission $e$ are represented by the function $\lambda g(e)$, with $\lambda>0$, and $g(e)$ increasing and concave: $g^{\prime}(e)>0$ and $g^{\prime \prime}(e)<0$ for all $e>0$. Parameter $\lambda$ introduces a simple way to parameterize the gains of the firm (due, for example, to cost reduction) from polluting. A firm with higher $\lambda$ is a firm whose private benefits from polluting are higher. ${ }^{5}$ To isolate effects and to concentrate on simple compliance problems, I assume that $\lambda$ is public information.

In order to control pollution, the regulator sets a standard $\epsilon_{\lambda}$ that may depend on the characteristics of the firm, $\lambda$, and that limits emissions. I suppose that the firms have the obligation to self-report their emissions. ${ }^{6}$

If the level of emissions is not perfectly and costlessly monitorable, then the auditing strategy of the enforcement agency ( $E A$ hereafter) and the reporting strategy of the firm (in addition to its emissions strategy) are strategic decisions. I denote by $\alpha$ the probability that the $E A$ will audit the emissions of the firm. I assume that the probability of being audited may depend on the firm's parameter $\lambda$ but is independent of the report made by the firm. ${ }^{7}$ When audited, the firm's true emissions are identified without error. ${ }^{8}$

The firm may choose a report $z$ that does not coincide with the true emission level $e$. Note however, that $e \geq \epsilon_{\lambda}$ since a firm never pollutes less than the standard and $z \leq e$

\footnotetext{
${ }^{5}$ For the purpose of the model, I concentrate on the decision of the firm concerning its true and reported levels of emission. If the product market is competitive, decisions on output are optimal. Hence, the abatement cost function accounts for optimal output adjustment. So, under perfect competition, there is no need to pay attention explicitly to the output market.

${ }^{6}$ Self-reporting is common in many set-ups where environmental regulation is based on standards. For example, in the U.S., all major sources (by Title V of the Clean Air Act) have to evaluate their own compliance status and submit certified reports.

${ }^{7}$ This is a sensible hypothesis. Moreover, Macho-Stadler and Pérez-Castrillo (2006), applying a set-up close to the one analyzed here to environmental taxes, show that restricting attention to this class of policy is without loss of generality in many scenarios.

${ }^{8}$ For a set-up where there is an exogenous probability of identifying the true emissions when audited, see Macho-Stadler and Pérez-Castrillo (2006). For models where this characteristic is a choice for the firm, see Malik (1990a) and Heyes (1994).
} 
since it never reports a higher emission level than the real one. When the firm reports a level of emissions higher than the standard, it has to pay a fine. For convenience, this fine is assumed to be linear. Hence, letting $\tau$ be the fine per unit of exess emissions, the total fine is given by $\tau \cdot\left(z-\epsilon_{\lambda}\right)$. Moreover, when the firm reports a level of emissions inferior to the real one, if it is audited and its true emission level is identified, then it pays a penalty associated with its underreporting. From now on I will refer to the difference between reported and true emissions as "evasion." The penalty takes the form of a function $\theta(e-z)$ increasing and convex in the level of evasion. In addition, we assume that $\theta(0)=0, \theta^{\prime}(0)>\tau$, and $\theta^{\prime \prime}(e-z)>0$ for $e>z .^{9}$

Therefore, the expected profits of a firm with parameter $\lambda$ facing an audit probability $\alpha$ and a standard $\epsilon_{\lambda}$, when it chooses an emission level $e$ and it reports $z$ can be written as:

$$
E \Pi\left(\lambda, \alpha, \epsilon_{\lambda} ; e, z\right)=\lambda g(e)-\tau .\left(z-\epsilon_{\lambda}\right)-\alpha \theta(e-z) \quad \text { for } e \geq \epsilon_{\lambda}, \epsilon_{\lambda} \leq z \leq e .
$$

The firm chooses the optimal levels $e^{o}$ and $z^{o}$ in order to maximize the expected profits (1). If the solution is interior, the first-order conditions are:

$$
\begin{gathered}
\frac{\partial E \Pi}{\partial e}=\lambda g^{\prime}(e)-\alpha \theta^{\prime}(e-z)=0, \\
\frac{\partial E \Pi}{\partial z}=-\tau+\alpha \theta^{\prime}(e-z)=0 .
\end{gathered}
$$

Note that the conditions characterizing the interior solution do not depend on $\epsilon_{\lambda}$.

The firm can always buy more emissions at the price $\tau$ (even if the audit probability is very high). The firm will choose to do so if $\lambda g^{\prime}\left(\epsilon_{\lambda}\right)>\tau$ and the solution is interior in $z$. For the sake of simplicity in presenting the results, I define $e_{\lambda}^{*}$ as the emissions under perfect monitoring $(\alpha=1)$. This level is the maximum between the standard and the

\footnotetext{
${ }^{9}$ The assumptions on $\theta$ are common in the literature. As to the comparison between $\tau$ and $\theta$, recall that different transgressions usually have different consequences. Assuming that $\theta^{\prime}(0)>\tau$ is according to stylized facts and common sense: The marginal penalty for a reported violation, reporting $z$ greater than $\epsilon_{\lambda}$, should be smaller than the marginal penalty for a detected unreported violation, $e$ greather than $z$. For example, for water pollution, Downing (1983) reports that the penalty for falsification of records is far greather than the penalty for violating the source operating standard. However, if this assumption does not hold, the corresponding model can be analyzed along the same lines without difficulties.
} 
level of emissions such that $\lambda g^{\prime}(e)=\tau$. Formally, $e_{\lambda}^{*}=\max \left\{\epsilon_{\lambda},\left(g^{\prime}\right)^{-1}(\tau / \lambda)\right\}$. Note that for a given standard $\epsilon_{\lambda}, e_{\lambda}^{*}$ is decreasing in $\tau$, until the point where $\tau=\lambda g^{\prime}\left(\epsilon_{\lambda}\right)$; from then on $e_{\lambda}^{*}=\epsilon_{\lambda}$.

Next, Proposition 1 establishes the optimal behavior of the firm:

Proposition 1 For a given standard $\epsilon_{\lambda}$, audit probability $\alpha$, fine rate $\tau$, and penalty function $\theta($.$) , the optimal emission and report decisions \left(e^{o}, z^{o}\right)$ for the firm with parameter $\lambda$ are:

(a) If $\alpha=0$, then $e^{o}$ grows unbounded and $z^{o}=\epsilon_{\lambda}$.

(b) If $\alpha \in\left(0, \frac{\min \left\{\tau, \lambda g^{\prime}\left(\epsilon_{\lambda}\right)\right\}}{\theta^{\prime}\left(e_{\lambda}^{*}-\epsilon_{\lambda}\right)}\right)$, then $e^{o}>e_{\lambda}^{*}$ as defined by (4) and $z^{o}=\epsilon_{\lambda}$ :

$$
\lambda g^{\prime}\left(e^{o}\right)-\alpha \theta^{\prime}\left(e^{o}-\epsilon_{\lambda}\right)=0
$$

(c) If $\alpha \in\left[\frac{\min \left\{\tau, \lambda g^{\prime}\left(\epsilon_{\lambda}\right)\right\}}{\theta^{\prime}\left(e_{\lambda}^{*}-\epsilon_{\lambda}\right)}, \frac{\min \left\{\tau, \lambda g^{\prime}\left(\epsilon_{\lambda}\right)\right\}}{\theta^{\prime}(0)}\right)$, then $e^{o}=e_{\lambda}^{*}$ and $z^{o} \in\left[\epsilon_{\lambda}, e_{\lambda}^{*}\right)$ as defined by $(5)$ :

$$
\tau=\alpha \theta^{\prime}\left(e_{\lambda}^{*}-z^{o}\right)
$$

(d) If $\alpha \geq \frac{\min \left\{\tau, \lambda g^{\prime}\left(\epsilon_{\lambda}\right)\right\}}{\theta^{\prime}(0)}$, then $e^{o}=e_{\lambda}^{*}$ and $z^{o}=e_{\lambda}^{*}$.

The solution in terms of emissions and reports as a function of the audit probability $\alpha$ is illustrated in Figures 1 and 2. In Figure 1, the fine rate $\tau$ is low and makes it optimal for the firm to buy extraemissions for frequent levels of audits. In other words, in Figure $1, e_{\lambda}^{*}=\left(g^{\prime}\right)^{-1}(\tau / \lambda)$. In Figure 2, the penalty rate $\tau$ is high enough, so that buying extra emissions is never attractive for the firm, hence $e_{\lambda}^{*}=\epsilon_{\lambda}$. In this case, since $\theta^{\prime}\left(e_{\lambda}^{*}-\epsilon_{\lambda}\right)=\theta^{\prime}(0)$, Region (c) does not exist. Note also that in Figure 1, if the penalty function $\theta($.$) is linear, then \theta^{\prime}(0)=\theta^{\prime}\left(e_{\lambda}^{*}-\epsilon_{\lambda}\right)$, and Region (c) also vanishes.

[Insert Figure 1 and Figure 2 about here]

Considering Proposition 1 , if the firm is not subject to any audit $(\alpha=0)$, thus not fearing any inspection, it pollutes freely while claiming to be compliant, that is, $e^{o}$ is the maximum one and the firm reports to be respectful of the standard: $z^{o}=\epsilon_{\lambda}$. As the audit pressure on the firm increases, the firm decreases its level of emissions, while still reporting that it is compliant with the standard. This is an important insight from the analysis of the model, also highlighted in Macho-Stadler \& Pérez-Castrillo (2006) for the 
case of environmental taxes: When auditing is not too frequent, its deterrence effect on emissions is much stronger than its effect on the report. ${ }^{10}$

When the audit pressure is strong, the firm chooses the "minimum" level of emissions $e_{\lambda}^{*}$ (the level that the firm would choose under perfect monitoring but that, depending on $\tau$, may or may not coincide with the standard $\epsilon_{\lambda}$ ) and also makes a report closer to the truth. This corresponds to Region (c), where there is an interior solution for both emissions and report. ${ }^{11}$ Finally, if the perceived audit pressure $\alpha$ is even stronger (Region (d)), the firm's decision is the same as under perfect monitoring, that is, $e^{o}=z^{o}=e_{\lambda}^{*}$. Note however that the emissions may be greater than the standard since $\tau$ allows the firm to buy more emissions.

With respect to the frequency of the audit, the comparative statics go on the intuitive direction:

- $e^{o}$ is non-increasing in $\alpha$ and

- $z^{o}$ is non-decreasing in $\alpha$.

The effects of the standard are more interesting:

- The firm's emissions are non-decreasing in the standard (they are increasing in Region (b), as (4) shows), and

- the report is non-increasing in $\epsilon_{\lambda}$.

In Figures 1 and 2 that means that an increase in the standard shifts function $e^{o}$ up. In addition, in Proposition 1, if $\lambda g^{\prime}\left(\epsilon_{\lambda}\right)>\tau$, then, for a given $\tau$ and $\theta($.$) , Region (b) grows$ and Region (c) shrinks when the standard $\epsilon_{\lambda}$ increases. Hence, for a given enforcement policy, the higher the standard the more the firm tends to pollute. The only exception is the case where $\theta$ is linear because then the equation defining the firm's effort (4) does not depend on the standard.

A final consideration is the role of self-reporting in this model. Self-reporting is proposed in environments where pollution is stochastic. ${ }^{12}$ In the model framework where

\footnotetext{
${ }^{10}$ Note that none of the results in this section depend on the objective function of the environmental agency. They are derived from the analysis of the optimal behavior of the firm. Also, as it will be clear later, this last result does not depend on the fine being linear while the penalty function is convex.

${ }^{11}$ This is the region where most papers concentrate their analysis.

${ }^{12}$ Harford (1987), Helland (1998), Innes (1999), Kaplow and Shavell (1994), Livernois and McKenna (1999), and Malik (1993), among others, have considered models where self-reporting is an important
} 
final pollution is a non-random variable, self-reporting does not fulfill this role. However, it is important to know its consequences. Without self-reporting the firm's expected profit becomes:

$$
E \Pi\left(\lambda, \alpha, \epsilon_{\lambda} ; e\right)=\lambda g(e)-\alpha \theta\left(e-\epsilon_{\lambda}\right) \quad \text { for } e \geq \epsilon_{\lambda}
$$

whose interior solution is defined by

$$
\frac{\partial E \Pi}{\partial e}=\lambda g^{\prime}(e)-\alpha \theta^{\prime}\left(e-\epsilon_{\lambda}\right)=0
$$

which coincides with (4).

In the absence of self-reporting, the results of the firm's emission level for a given enforcement policy will coincide with the results when $e=\epsilon_{\lambda}$, as presented in Figure 2 . Hence, for a given standard $\epsilon_{\lambda}$, self-reporting does not affect a firm's emission level if selfreporting cannot be used to buy extra emissions (in other words, if $\tau$ is high enough), and alternatively, self-reporting leads to higher pollution levels when it allows to buy extra emissions.

\section{Choice of instruments}

In this Section I compare the outcome that an EA can implement when using three instruments to enforce the total emissions level: (a) standards combined with self-reporting, (b) environmental taxes, and (c) tradable permits.

\subsection{Environmental Taxes vs Standards}

First, note that if $t$ denotes the environmental taxes and $\theta$ denotes the penalty function, the expected profit of a firm with parameter $\lambda$ is:

$$
E \Pi(\lambda, \alpha, t ; e, z)=\lambda g(e)-t z-\alpha \theta(e-z) \quad \text { for } e \geq 0,0 \leq z \leq e .
$$

element in enforcement policies. It has been shown that self-reporting is a useful tool when pollution is stochastic. Allowing the firm to report the bad state of nature when damages ex-post are higher than expected reduces monitoring costs. Livernois and McKenna (1999) take the deterministic view and analyze a repeated game. 
Comparing (1) with (6), it can be checked that they coincide with each other when $\tau=t$ and $\epsilon_{\lambda}=0$. The question is then whether $\epsilon_{\lambda}=0$ is optimal (assuming that environmental taxes $t$ can be set equal to the fine $\tau$ ). Given the discussion in the static comparative on $\epsilon_{\lambda}$ done in the previous section, the answer is that with the same $\alpha$, the $E A$ is able to induce a lower level of pollution with taxes $\left(\epsilon_{\lambda}=0\right)$ than with standards $\left(\epsilon_{\lambda}>0\right)$. Note that this is also the case if $\tau$ is very high (in such a way that the firm does not buy extra emissions via this channel) and $\epsilon_{\lambda}>0$, with $t=\lambda g^{\prime}\left(\epsilon_{\lambda}\right)$. In this situation, under full compliance both instruments imply the same emissions level, but the monitoring cost necessary to achieve full compliance with standards is higher than with taxes. Intuitively, standards reduce the size of the penalty because they increase the minimum report firms may do without cost (the report is at least equal to the standard). Only when the penalty function $\theta$ is linear do total emissions not depend on the minimal report, and then taxes and standards lead to the same results. The previous result is summarized in Corollary 1:

Corollary 1 When the only objective of the EA is to minimize total emissions at the minimum costs, then environmental taxes are at least as effective as standards, and they are strictly superior if the penalty function $\theta$ is strictly convex.

\subsection{Optimal Policy under Environmental Taxes}

To be able to compare environmental taxes and permits, I briefly present here the optimal audit policy under taxes. I consider an $E A$ that wants to minimize total emissions, ${ }^{13}$ when it faces a population of firms parameterized by $\lambda$, distributed over the interval $[\underline{\lambda}, \bar{\lambda}]$, $0<\underline{\lambda}<\bar{\lambda}$ according to the density function $f(\lambda)$, with $f(\lambda)>0$ for all $\lambda \in[\underline{\lambda}, \bar{\lambda}]$. The $E A$ can devote a fixed budget $B$ to auditing; thus I normalize the cost of one audit to 1 .

\footnotetext{
${ }^{13}$ In assuming that the objective of the enforcement agency is to achieve the highest level of compliance given its enforcement budget and not to raise money, I follow many other papers, for example, Garvie and Keeler (1994) and Macho-Stadler and Pérez-Castrillo (2006).
} 
The optimal monitoring policy $(\alpha(\lambda))_{\lambda \in[\underline{\lambda}, \bar{\lambda}]}$ is the solution to the following program:

$$
\begin{gathered}
\operatorname{Min}_{\int_{\underline{\lambda}}}^{\bar{\lambda}} e(\lambda) f(\lambda) d \lambda \\
\text { s.t. } \int_{\underline{\lambda}}^{\bar{\lambda}} \alpha(\lambda) f(\lambda) d \lambda \leq B \\
e(\lambda) \in \operatorname{argmax} E \Pi(\lambda, \alpha(\lambda), t, \theta ; e, z),
\end{gathered}
$$

where $E \Pi($.$) are the expected profits defined in (6). In the results, e_{\lambda}^{*}$ denotes the emissions level of a firm of characteristic $\lambda$ that fully complies with the pollution objective (which is defined by $\lambda g^{\prime}\left(e_{\lambda}^{*}\right)=t$ ), and $\bar{B}$ denotes the budget necessary to achieve full compliance in the population of firms (obviously, the budget $\bar{B}$ depends on the characteristics of the firms' population).

Proposition 2 (Macho-Stadler and Pérez-Castrillo, 2006) The optimal monitoring policy as a function of the budget $B$ is:

(i) When $B>\bar{B}$, the $E A$ chooses an audit policy that implies $\alpha(\lambda) \geq \frac{t}{\theta^{\prime}\left(e_{\lambda}^{*}\right)}$, for all $\lambda \in[\underline{\lambda}, \bar{\lambda}]$. Firms' emission levels are $e^{o}(\lambda)=e_{\lambda}^{*}$.

(ii) When $B \leq \bar{B}$, there exists $\lambda(B)$, with $\underline{\lambda} \leq \lambda(B) \leq \bar{\lambda}$, such that

(ii.I) For firms with $\lambda \geq \lambda(B)$, then $\alpha(\lambda)=\frac{t}{\theta^{\prime}\left(e_{\lambda}^{*}\right)}$. A firm's emission level is $e^{o}(\lambda)=$ $e_{\lambda}^{*}$

(ii.II) For firms with $\lambda<\lambda(B)$, then $\alpha(\lambda)>0$. A firm's emission level $e^{o}(\lambda)>e_{\lambda}^{*}$ is increasing in $\lambda$.

An important feature of the result presented in Proposition 2 is that, except if $B$ is very high, in equilibrium $\alpha(\lambda) \leq \frac{t}{\theta^{\prime}\left(e_{\lambda}^{*}\right)}$, which implies that, being concerned only about emissions, the $E A$ never induces a firm to report more than $z=0$ (or the lowest possible report). That is, the optimal audit policy induces all firms to report not having polluted (or the minimum credible report). ${ }^{14}$ Also the $E A$ biases its strategy against those firms that value pollution more.

It can be shown that $\lambda(B)$ is decreasing with $B$. If $B$ is very high, for $B=\bar{B}$, $\lambda(B)=\bar{\lambda}$, while if $B$ is very low, then $\lambda(B)=\underline{\lambda}$. Obviously, the optimality of the

\footnotetext{
${ }^{14}$ Macho-Stadler and Pérez-Castrillo (2006) also show that for the optimal monitoring device, penalty revenues are maximal (this may be used to provide incentives to the $E A$ ).
} 
proposed audit policy relies very much on the fact that the $E A$ can announce and commit on the audit strategy before the firms make any decision. The main objective of the policy is to deter firms from polluting, and dissuasion plays an important role. ${ }^{15}$ The possibility for firms to underreport their emission levels (and the cost and difficulty of monitoring them) leads to actual emission levels higher than those that would take place under full compliance.

\subsection{Tradable Permits vs Environmental Taxes}

When the environmental regulation is based on tradable permits, the regulator distributes a number of permits $L$ among the firms. Those permits can be traded in a (by assumption perfectly competitive) market that determines a price $p .{ }^{16}$ The price $p$ represents either the price that a firm pays for a permit, or the outside option for a permit that the firm does not sell. Hence, independent of the initial distribution of permits, if $z$ denotes the number of permits that a firm holds at the end, then the firm's expected profits (up to a constant) can be writen as

$$
E \pi(e, z)=\lambda g(e)-p z-\alpha \theta(e-z)
$$

The conclusions about the firm's behavior when confronted with an audit probability $\alpha$ and a penalty function $\theta(e-z)$ are very close to the ones presented before (with the exception that now $p$ is endogenous at the aggregate level and depends on the total number of permits).

This paper finds that environmental taxes are also superior to tradable permits. To see why, consider two situations, one with a tax rate $t$ and another where, given the number of permits, the market price for them is $p=t$. To see that the $E A$ with a given $B$

\footnotetext{
${ }^{15}$ If the auditing is decided simultaneously with or after the firms have polluted and reported, dissuasion is less effective and the equilibrium emerging in this game is different (see, for example, Franckx, 2002).

${ }^{16}$ The effects of noncompliance on the performance of tradable permits has been studied by Malik (1990b), and Stranlund and Dhanda (1999). The results in the previous papers do not coincide with the one presented here because the first author assumes that the (exogenous) probability of an audit is increasing on the firm's emissions and the second authors concentrate on the interior solution of the firm's program. Hence, they conclude that emissions are independent of the audit probability and penalty pressure.
} 
achieves a lower level of emissions under a tax system than under a permit system, take the function $\widetilde{\alpha}(\lambda)$ that is optimal for $E A$ in the case of permits. In this situation, some firms will buy the $L$ permits (if it is not the case, in a competitive market, the price of the permit will go to zero), which implies $z>0$ for some firms. Take the same monitoring policy, and apply it to a situation based on a tax $t=p$. Then the firms will report the same level of emissions as in the case of permits, in particular some of them will chose $z>0$. This implies that for the case of taxes, $\widetilde{\alpha}(\lambda)$ is too high of an audit pressure for these firms. Given the $E A$ objective, it is optimal to induce firms to report zero. Hence, I can conclude that

Corollary 2 When the only objective of the EA is to minimize total emissions at minimum costs, then environmental taxes are at least as effective as tradable permits.

\subsection{Efficiency}

In this study, the distribution of emissions levels $e_{\lambda}$ for the population of $\lambda$ is (static) efficient if for this distribution, the marginal benefits for all forms are equal. In other words, the distribution of emissions maximizes total firm benefits. Then, in this section I will show that overpollution is not the only negative consequence of imperfect compliance. It also destroys the property of (static) efficiency, at least if the audit policy is designed to minimize total emissions. I explain here the distortion for the case of taxes.

If a uniform tax $t$ is set, then under full compliance the marginal costs from emissions are equal for all firms. Indeed, a firm with parameter $\lambda$ chooses its emission level $e_{\lambda}^{*}$ such that $\lambda g^{\prime}\left(e_{\lambda}^{*}\right)=t$, and there is no room to distribute total emissions $\int_{\underline{\lambda}}^{\bar{\lambda}} e_{\lambda}^{*} f(\lambda) d \lambda$ in a more efficient way. Hence, full compliance leads to efficiency. In contrast, under imperfect compliance, the differences in firms' characteristics lead to different incentives to underreport and also to different pressure levels from the $E A$. To illustrate this point, I present an example (that allows to obtain explicit solutions) where $g(e)=\frac{1}{a} e^{a}$, with $a<1$, and $\theta(x)=\theta x$. Moreover, I consider that the distribution function of $\lambda$ is uniform in the interval $(0,1]$.

To study the efficiency aspect, I will proceed in three steps. First, compute the optimal audit policy for the economy, i.e., the function $\alpha(\lambda)$ that minimizes total emissions. Then, 
given the function $\alpha(\lambda)$, compute (for given particular parameters) the emissions level for every firm. Second, add them up to find the total emissions in the economy when the optimal monitoring policy is implemented. Given the total emissions computed before, I find the distribution of emissions among the firms that is efficient.

\subsubsection{Optimal monitoring policy and firms' emissions at equilibrium ${ }^{17}$}

With the functional forms of this example, the bound $\bar{B}$ identified in Proposition 2 is $\bar{B}=\frac{t}{\theta}$. Define $\widehat{B} \equiv \frac{(2-a) t}{(3-a) \theta}$ and

$$
\begin{array}{ll}
\beta=\frac{(2-a)^{\frac{2-a}{1-a}}}{(1-a)(3-a)^{\frac{2-a}{1-a}} \theta^{\frac{1}{1-a}} B^{\frac{2-a}{1-a}}} & \text { when } B<\widehat{B}, \text { and } \\
\beta=\frac{(3-a)^{\frac{1}{1-a}} \theta^{\frac{2-a}{1-a}}}{(1-a) t^{\frac{3-a}{1-a}}}\left[\frac{t}{\theta}-B\right]^{\frac{1}{1-a}} \quad \text { when } B \in[\widehat{B}, \bar{B}) .
\end{array}
$$

Also, define

$$
\begin{aligned}
& \lambda(B)=1 \quad \text { when } B<\widehat{B} \\
& \lambda(B)=\frac{(1-a)^{1-a} t^{2-a} \beta^{1-a}}{\theta^{1-a}} \quad \text { when } B \in[\widehat{B}, \bar{B}) \\
& \lambda(B)=0 \quad \text { when } B \geq \bar{B} \text {. }
\end{aligned}
$$

The solution to the Program (7) is

(i) For $\lambda \leq \lambda(B)$,

which leads to

$$
\alpha_{\lambda}=\frac{\lambda^{\frac{1}{2-a}}}{(1-a)^{\frac{1-a}{2-a}} \theta^{\frac{1}{2-a}} \beta^{\frac{1-a}{2-a}}},
$$

$$
e_{\lambda}^{o}=\frac{\lambda^{\frac{1}{2-a}}(1-a)^{\frac{1}{2-a}} \beta^{\frac{1}{2-a}}}{\theta^{\frac{1}{2-a}}}>e_{\lambda}^{*} .
$$

(ii) For $\lambda>\lambda(B)$,

$$
\alpha_{\lambda}=\frac{t}{\theta} \quad \text { and } \quad e_{\lambda}^{o}=e_{\lambda}^{*}=\frac{\lambda^{\frac{1}{1-a}}}{t^{\frac{1}{1-a}}} .
$$

For $B<\widehat{B}$, the optimal policy is of the first type for all firms, which leads all firms to emissions higher than under full compliance. For $B \in[\widehat{B}, \bar{B})$ there are firms compliant and others non compliant. For $B \geq \bar{B}$, then $\lambda(B)=0$ and all firms are compliant in terms of pollution level.

\footnotetext{
${ }^{17}$ See the Appendix for the details of the computation.
} 


\subsubsection{Total emissions and efficient distribution of emissions}

To study the efficiency aspect, denote by $E$ the total level of emissions:

$$
E \equiv \int_{\lambda} e_{\lambda} f_{\lambda} d \lambda
$$

Denote by $e_{\lambda}^{F}$ the efficient distribution of emissions $E$, that is the distribution that maximizes total profits subject to the constraint that total emissions are equal to $E$.

(a) Consider the case: $a=1 / 2, t=1, \theta=1$, and $B=2 / 5$. This parameter combination leads all firms to pollute more than $e_{\lambda}^{*}$. More precisely, the variables take the values:

\begin{tabular}{|l|l|l|l|l|l|}
\hline$\widehat{B}$ & $\beta$ & $e_{\lambda}^{o}$ & $e_{\lambda}^{*}$ & $E$ & $e^{F}$ \\
\hline $3 / 5$ & $27 / 4$ & $\frac{9}{8} \lambda^{\frac{2}{3}} \sqrt[3]{8}$ & $\lambda^{2}$ & $27 / 20$ & $\frac{81}{20} \lambda^{2}$ \\
\hline
\end{tabular}

Plotting functions $e_{\lambda}^{o}, e_{\lambda}^{*}$, and $e^{F}$ produces Figure 3 .

[Insert Figure 3 about here]

(b) Consider now the case: $a=1 / 2, t=1, \theta=1$, and $B=7 / 10$. Here the budget is higher and both regions exist: some firms, the ones with lower $\lambda$, choose in equilibrium $e_{\lambda}^{o}>e_{\lambda}^{*}$ and those with lower $\lambda$ set $e_{\lambda}^{o}=e_{\lambda}^{*}$. The variables take the values:

\begin{tabular}{|l|l|l|l|l|l|l|}
\hline$\widehat{B}$ & $\beta$ & $e_{\lambda}^{o}$ & $e_{\lambda}^{*}$ & $\lambda(B)$ & $E$ & $e^{F}$ \\
\hline $3 / 5$ & $9 / 8$ & $\frac{3}{8} \lambda^{\frac{2}{3}} \sqrt[3]{6}$ & $\lambda^{2}$ & $\frac{3}{4}$ & $\frac{107}{240}$ & $\frac{107}{80} \lambda^{2}$ \\
\hline
\end{tabular}

Then, plotting the functions $e_{\lambda}^{o}, e_{\lambda}^{*}$, and $e_{\lambda}^{F}$ produces Figure 4.

[Insert Figure 4 about here]

Figures 3 and 4 show that the efficient distribution of total emissions $E$ does not coincide with the distribution of emissions induced by the optimal monitoring policy when this policy is designed to minimize total emissions. Firms with a lower $\lambda$ are induced to pollute more than the efficient level, while firms with a high $\lambda$ are induced to pollute less (the cut-off values being $\lambda=0.64$ in Figure 3 and $\lambda=0.60$ in Figure 4 ). If the $E A$ 's only concern is to preserve the (static) efficiency property of firms' emissions distribution, the monitoring policy will allocate the budget in such a way that firms' aggregate profits ares maximized, but then total emissions will be higher. 
If both total emissions and efficiency are an issue, then under imperfect compliance, in designing the monitoring policy the $E A$ has to consider the trade-off between minimizing global emissions and inducing firms' emissions to satisfy the (static) efficiency property. There is no way both objectives can be fulfilled at the same time, unless if the budget is large enough to induce all the firms to choose the full-compliance emissions level.

\section{Final remarks}

This paper aims to present a better understanding of he role of imperfect compliance in environmental regulation. The results predict first that, when facing a population of heterogeneous firms, an $E A$ will be more effective in reducing total emissions if the regulatory instrument is based on taxes than if the instrument is based on standards or permits.

Second, the optimal auditing policy may very well lead to a reasonable level of emissions, coupled with a high level of environmental tax evasion. The reason is simple: since the ultimate goal of enforcement agencies is environmental quality, not tax revenue, their best policy leads firms to comply with the environmental objective but to disregard the problem of environmental tax evasion. Finally, we should expect that firms' emissions decisions are not expected to satisfy the efficiency property.

These results complement those obtained in Macho-Stadler and Pérez-Castrillo (2006) and stand in contrast to some of the conclusions of the previous literature. Under full compliance (and certainty), both taxes and permits lead to an efficient distribution of total emissions, given the equality of marginal costs of emissions across firms. Standards can also reach the same efficiency result if the $E A$ can set the adequate quota for each firm. (The advantage of taxes and permits is that they reach the same result with simpler regulatory design.) Also under imperfect compliance, Sandmo (2002) (following Harford, 1978 and 1987) finds that the efficiency property of taxes and standards (with self-reporting) continues to hold for the case of a fixed monitoring probability (a given $\alpha$ ) and also when the probability depends only on the extent of underreporting (i.e., the probability takes the form of an increasing function $\alpha(e-z))$. The reason for this conclusion is that he concentrates on monitoring probabilities that lead to interior solutions (that is, Region 
(c) in Proposition 2). ${ }^{18}$ As this paper proves, when the $E A$ is concerned only about total emissions, the optimal audit policy in fact leads firms to pick corner solutions. Therefore, under imperfect compliance the efficiency property is no longer satisfied for any environmental instrument, unless the budget is very large.

The result that using taxes allows the $E A$ to reach a lower level of total pollution than using standards or permits also comes from the analysis of the corner solutions. Otherwise, Harford (1987) shows the similarity between taxes and standards combined with selfreporting. A different comparison of instruments is made in Keeler (1991), who finds that noncompliance can be more important under permits than under a system of uniform standards in a model where firms are differentiated by their pollution abatement costs and where monitoring probabilities and penalty functions are exogenous and constant across firms. In a similar vein, Malik (1992) finds that tradable permits may be more costly to enforce than uniform emission standards.

In the model, I concentrate on a particular set-up with many firms and non-random emissions, where the gains from emissions are known by the regulator. There are many markets where the characteristics of the emissions, the information, and even the market structure, are quite different from the previous ones. Analyzing the effects of noncompliance in other settings would be an interesting line for future research. In particular the study of how to adapt policy instruments to the particular emissions, considering the enforceability issue is still an open and a key question for environmental regulation.

Another interesting line of research is to take into serious consideration how differences between countries lead to the use of different instruments. Each country is characterized by a set of institutions, a certain capacity and effectiveness of monitoring techniques, and a production and environmental culture. All of these aspects may play a crucial role in the choice of environmental instruments. ${ }^{19}$

A related issue for study is the incentives to monitor and the behavior of firms in

\footnotetext{
${ }^{18}$ Sandmo (2002) shows that the previous results still hold when firms are risk-averse.

${ }^{19}$ For example, in Germany, Sweden, and the Netherlands, of the 151 instruments in use for environmental protection, approximately half are charges and one-third are subsidies. Other instruments used are deposit-refund systems or permits. In the developing world property rights are used more often, and they seem to play a particularly important role, for example, in protecting the forestland and preventing its conversion to other uses (for example, in Papua, New Guinea).
} 
situations where the pollution policy and/or its enforcement are in the hands of several bodies: international organizations, each country's government, local governments and municipalities. The different levels may have different objectives and concerns, and the enforcement issue may be affected.

\section{Appendix}

Proof of Proposition 1. I distinguish two cases. When $\tau<\lambda g^{\prime}\left(\epsilon_{\lambda}\right)$, then the optimal emissions decision is always given by (2), that is, $e^{o}$ is always interior. In this case, the proof goes parallel to the proof of Proposition 1 in Macho-Stadler \& Pérez-Castrillo (2006). The same analysis holds when $\tau=\lambda g^{\prime}\left(\epsilon_{\lambda}\right)$.

When $\tau>\lambda g^{\prime}\left(\epsilon_{\lambda}\right)$, then equations (2) and (3) cannot hold simultaneously. Given that (2) cannot hold without (3), the optimal report is $z^{o}=\epsilon_{\lambda}$. Now, if $\alpha=0$, that is, in Region $(a)$, the firm chooses the maximum $e^{o}$. For $\alpha>0$, an interior solution in $e$ exists if and only if $\lambda g^{\prime}\left(\epsilon_{\lambda}\right)<\alpha \theta^{\prime}(0)$. This corresponds to the solution in Region (b). In the other case, which corresponds to Region $(d), e^{o}=\epsilon_{\lambda}$, which also corresponds to $e_{\lambda}^{*}$. Region $(c)$ does not exist when $\tau>\lambda g^{\prime}\left(\epsilon_{\lambda}\right)$ given that $e_{\lambda}^{*}-\epsilon_{\lambda}=0$.

Proof. of the Example.

From Proposition 2, at the optimum and when $B$ is not very high, the optimal inspection policy leads to $z_{\lambda}=0$ for all $\lambda$. This is equivalent to the constraint that $\alpha_{\lambda}$ cannot be larger than $\frac{t}{\theta^{\prime}\left(e_{\lambda}^{*}\right)}$, where $e_{\lambda}^{*}$ is defined by $\lambda g^{\prime}\left(e_{\lambda}^{*}\right)=t$. Then, the $E A$ solves the following program:

$$
\begin{gathered}
\operatorname{Min}_{e_{\lambda}, \alpha_{\lambda}} \int_{\lambda} e_{\lambda} f_{\lambda} d \lambda \\
\text { s.t. } \int_{\lambda} \alpha_{\lambda} f_{\lambda} d \lambda=B(\beta) \\
\lambda g^{\prime}\left(e_{\lambda}\right)-\alpha_{\lambda} \theta^{\prime}\left(e_{\lambda}\right)=0 \text { for all } \lambda\left(\eta_{\lambda}\right) \\
\alpha_{\lambda} \leq \frac{t}{\theta^{\prime}\left(e_{\lambda}^{*}\right)} \text { for all } \lambda\left(\varkappa_{\lambda}\right) .
\end{gathered}
$$

I denote respectively by $\beta, \eta_{\lambda}$, and $\varkappa_{\lambda}$ the Lagrange multiplier of the constraint of the same name. In the example, $g^{\prime}(e)=e^{a-1}, g^{\prime \prime}(e)=-(1-a) e^{a-2}, \theta^{\prime}(x)=\theta$ and $\theta^{\prime \prime}(x)=0$. 
Then, the first order copnditions of the previous program are:

$$
\begin{gathered}
\frac{\partial L}{\partial e_{\lambda}}=f_{\lambda}-\eta_{\lambda} \lambda(1-a) e_{\lambda}^{a-2}=0 \\
\frac{\partial L}{\partial \alpha_{\lambda}}=\beta f_{\lambda}-\eta_{\lambda} \theta+\varkappa_{\lambda}=0 .
\end{gathered}
$$

I use constraint $\left(\eta_{\lambda}\right)$ to get:

$$
\alpha_{\lambda}=\frac{\lambda e_{\lambda}^{a-1}}{\theta}
$$

Region $1: e_{\lambda}>e_{\lambda}^{*}=\frac{\lambda^{\frac{1}{1-a}}}{t^{1-a}}$. Hence, $\varkappa_{\lambda}=0$. The FOCs are written as:

$$
f_{\lambda}=\eta_{\lambda}\left[\lambda(1-a) e_{\lambda}^{a-2}\right]
$$

and

$$
\beta f_{\lambda}=\eta_{\lambda} \theta
$$

From (12) and (13):

$$
\frac{1}{\beta}=\frac{\lambda(1-a) e_{\lambda}^{a-2}}{\theta}
$$

Equation (14) defines $e_{\lambda}$ as a function of $\beta$ :

$$
e_{\lambda}=\frac{\lambda^{\frac{1}{2-a}}(1-a)^{\frac{1}{2-a}} \beta^{\frac{1}{2-a}}}{\theta^{\frac{1}{2-a}}} .
$$

In this region,

$$
\alpha_{\lambda}=\frac{\lambda e_{\lambda}^{a-1}}{\theta}=\frac{\lambda^{\frac{1}{2-a}}}{(1-a)^{\frac{1-a}{2-a}} \theta^{\frac{1}{2-a}} \beta^{\frac{1-a}{2-a}}} .
$$

A necessary condition for the $e_{\lambda}$ to be a candidate solution is that this candidate must be such that $e_{\lambda} \geq e_{\lambda}^{*}$. Since the right-hand side of (14) is decreasing in $e_{\lambda}$, the necessary condition is:

$$
\frac{\lambda(1-a) e_{\lambda}^{* a-2}}{\theta} \geq \frac{1}{\beta}
$$

i.e.,

$$
\lambda \leq \frac{(1-a)^{1-a} t^{2-a} \beta^{1-a}}{\theta^{1-a}} .
$$

Region $2: \varkappa_{\lambda}>0$. Hence, $\alpha_{\lambda}=\frac{t}{\theta^{\prime}\left(e_{\lambda}^{*}\right)}$ and $e_{\lambda}=e_{\lambda}^{*}$. In this case, using sequentially (10) and (9) we obtain:

$$
\varkappa_{\lambda}=-\beta f_{\lambda}+\eta_{\lambda} \theta=-\beta f_{\lambda}+\frac{f_{\lambda}}{\lambda(1-a) e_{\lambda}^{a-2}} \theta
$$


The necessary condition for $e_{\lambda}^{*}$ to be a candidate is $\varkappa_{\lambda} \geq 0$, i.e.,

$$
\frac{\lambda(1-a) e_{\lambda}^{a-2}}{\theta} \leq \frac{1}{\beta}
$$

i.e.,

$$
\lambda \geq \frac{(1-a)^{1-a} t^{2-a} \beta^{1-a}}{\theta^{1-a}} .
$$

In this region,

$$
\alpha_{\lambda}=\frac{\lambda e_{\lambda}^{* a-1}}{\theta}=\frac{t}{\theta}
$$

The parameter $\beta$ is characterized by the budget constraint. When $\beta$ is very large (i.e., $B$ is small), the only region that exists is Region 1. In this case (also using the assumption that the distribution function is uniform), the budget constraint is:

$$
\frac{1}{(1-a)^{\frac{1-a}{2-a}} \theta^{\frac{1}{2-a}} \beta^{\frac{1-a}{2-a}}} \int_{0}^{1} \lambda^{\frac{1}{2-a}} d \lambda=B
$$

i.e.,

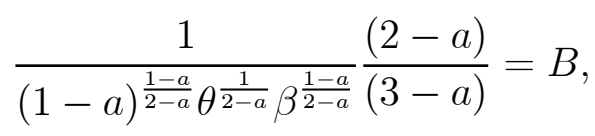

which gives,

$$
\beta=\frac{(2-a)^{\frac{2-a}{1-a}}}{(1-a)(3-a)^{\frac{2-a}{1-a}} \theta^{\frac{1}{1-a}} B^{\frac{2-a}{1-a}}} .
$$

This value for $\beta$ is indeed large enough so that only Region 1 exists if and only if equation (16) holds for the highest value of $\lambda$, that is, for $\lambda=1$. Hence, the condition is:

$$
\theta^{1-a} \leq(1-a)^{1-a} t^{2-a} \beta^{1-a}
$$

which, given the value for $\beta$, corresponds to:

$$
\theta^{1-a} \leq t^{2-a} \frac{(2-a)^{2-a}}{(3-a)^{2-a} \theta B^{2-a}}
$$

that is,

$$
B \leq \widehat{B} \equiv \frac{(2-a) t}{(3-a) \theta}
$$

Therefore, when $B \leq \widehat{B}$, the level of emission of a firm with parameter of efficiency $\lambda$ is given by (15), where $\beta$ is defined in (18). If $B>\widehat{B}$, then the budget constraint is written as:

$$
\frac{1}{(1-a)^{\frac{1-a}{2-a}} \theta^{\frac{1}{2-a}} \beta^{\frac{1-a}{2-a}}} \int_{0}^{\frac{(1-a)^{1-a} t^{2-a} \beta^{1-a}}{\theta^{1-a}}} \lambda^{\frac{1}{2-a}} d \lambda+\frac{t}{\theta} \int_{\frac{(1-a)^{1-a} t^{2-a} \beta^{1-a}}{\theta^{1-a}}}^{1} d \lambda=B
$$


and we obtain,

$$
\beta=\frac{(3-a)^{\frac{1}{1-a}} \theta^{\frac{2-a}{1-a}}}{(1-a) t^{\frac{3-a}{1-a}}}\left[\frac{t}{\theta}-B\right]^{\frac{1}{1-a}} .
$$

Note that for very high $B$, all firms will emit $e_{\lambda}^{*}$. This happens when the Lagrange multiplier of the budget constraint is zero, that is, when $B \geq \frac{t}{\theta}$.

Therefore, when $B \leq\left[\widehat{B}, \frac{t}{\theta}\right]$, the level of emission of a firm with parameter of efficiency $\lambda \leq \frac{(1-a)^{1-a} t^{2-a} \beta^{1-a}}{\theta^{1-a}}$ is given by (15), where $\beta$ is defined in (19) while the level of emissions of a firm with parameter $\lambda>\frac{(1-a)^{1-a} t^{2-a} \beta^{1-a}}{\theta^{1-a}}$ is $e_{\lambda}^{*}=\frac{\lambda^{\frac{1}{1-a}}}{t^{1-a}}$.

\section{References}

[1] Becker, G. (1968) Crime and Punishment: An Optimal Approach. Journal of Political Economy 76: 169-217.

[2] Cohen, M.A. (1999) Monitoring and Enforcement of Environmental Policy. In: Folmer H. and Tiltenberg T. (eds) The International Year-book of Environmental Resource Economics 1999/2000. Edward Elgar Publishing.

[3] Cohen M.A. (2000) Empirical Research on the Deterrent Effect of Environmental Monitoring and Enforcement. Environmental Law Institute. News and Analysis 30: 10245-10252.

[4] Cropper, M.L., Oates, W.E. (1992) Environmental Economics: A Survey. Journal of Economic Literature 30: 675-740.

[5] Dasgupta, S., Laplante, B., Maningi, N., Wang, H. (2001) Inspections, Pollution Prices, and Environmental Performance: Evidence from China. Ecological Economics 36: $487-498$.

[6] Downing, P. (1983) Bargaining in Pollution Control. Policy Study Journal 11: 577-86.

[7] Foulon, J., Lanoie, P., Laplante B. (2002) Incentives for Pollution Control: Regulation or Information?. Journal of Environmental Economics and Management 44: 169-187. 
[8] Franckx, L. (2002) The use of Ambient Inspections in Environmental Monitoring and Enforcement when the Inspection Agency Cannot Commit itself to Announced Inspection Probabilities. Journal of Environmental Economics and Management 43: 71-92.

[9] Garvie, D., Keeler, A. (1994) Incomplete Enforcement with Endogenous Regulatory Choice. Journal of Public Economics 55: 141-162.

[10] Harford, J. D. (1978) Firm Behavior under Imperfectly Enforceable Pollution Standards and Taxes. Journal of Environmental Economics and Management 5: 26-43.

[11] Harford, J. D. (1987) Self-reporting of Pollution and the Firm's Behavior under Imperfectly Enforceable Regulation. Journal of Environmental Economics and Management 14: 293-303.

[12] Helland, E. (1998) The Enforcement of Pollution Control Laws: Inspection, Violations and Self-reporting. Review of Economics and Statistics 80: 141-153.

[13] Heyes, A. (1994) Environmental Enforcement when "Inspectability" is Endogenous: A Model with Overshooting Properties. Environmetal Resource Economics 4: 479494.

[14] Heyes, A. (2000) Implementing Environmental Regulation: Enforcement and Compliance. Journal of Regulatory Economics 17: 107-129.

[15] Innes R. (1999) Remediation and Self-reporting in Optimal Law Enforcement. Journal of Public Economics 72: 379-393.

[16] Keeler, A. (1991) Noncompliant Firms in Transferable Discharge Permit Markets: some Extensions. Journal of Environmental Economics and Management 21: 180189.

[17] Kaplow, L., Shavell, S. (1994) Optimal Law Enforcement with Self-reporting Behavior. Journal of Political Economics 102: 583-606.

[18] Livernois, J., McKenna, C.J. (1999) Trust or Consequences. Enforcing Pollution Standards with Self-reporting. Journal of Public Economics 71: 415-440. 
[19] Macho-Stadler, I., Pérez-Castrillo, D. (2006) Optimal Enforcement Policy and Firms' Emissions and Compliance with Environmental Taxes. Journal of Environmental Economics and Management 51: 110-131.

[20] Malik, A. (1990a) Avoidance, Screening and Regulatory Enforcement. Rand Journal of Economics 21: 341-353.

[21] Malik, A. (1990b) Markets for Pollution Control when Firms are noncompliant. Journal of Environmental Economics and Management 18: 97-106.

[22] Malik, A. (1992) Enforcement Costs and the Choice of Policy Instruments for Pollution Control. Economic Inquiry 30: 714-721.

[23] Malik, A. (1993) Self-reporting and the Design of Policies for Regulating Stochastic Pollution. Journal of Environmental Economics and Management 24: 241-257.

[24] Montero, J.-P. (2002) Prices versus Quantities with Incomplete Enforcement. Journal of Public Economics 85: 435-454.

[25] Polinsky, A.M., Shavell, S. (2000) The Economic Theory of Public Enforcement of the Law. Journal of Economic Literature 38: 45-76.

[26] Sandmo, A. (2000) The Public Economics of Environment. Oxford University Press.

[27] Sandmo, A. (2002) Efficient Environmental Policy with Imperfect Compliance. Environmental Resource Economics 23: 85-103.

[28] Stranlund, J., Dhanda, K.K. (1999) Endogenous Monitoring and Enfrocement of a Transferable Emissions Permit System. Journal of Environmental Economics and Management 38: 267-282.

[29] Weitzman, M. (1974) Prices vs Quantities. Review of Economic Studies 41: 477-491. 


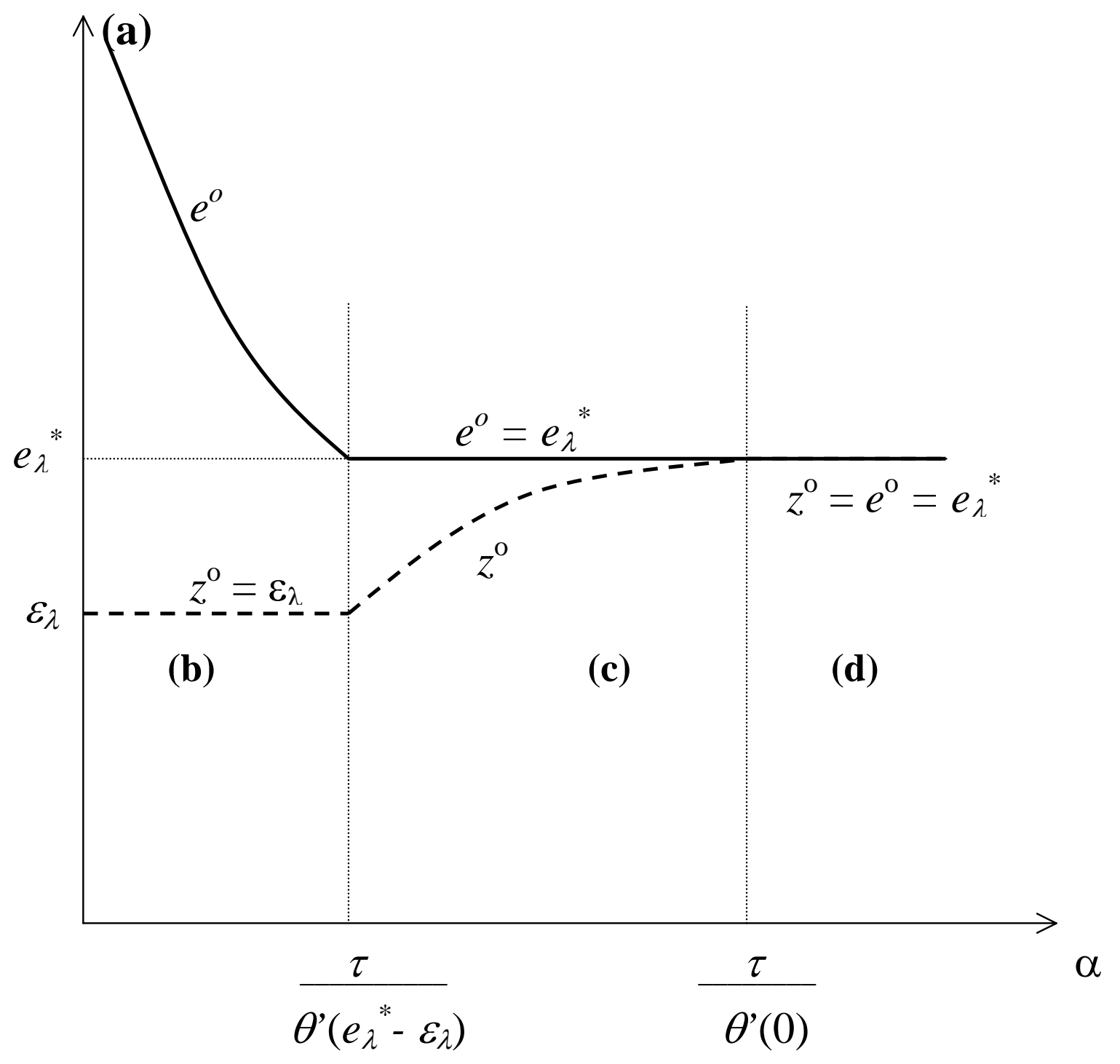

Figure 1: Firm's decisions for $e_{\lambda}{ }^{*}>\varepsilon_{\lambda}$ 


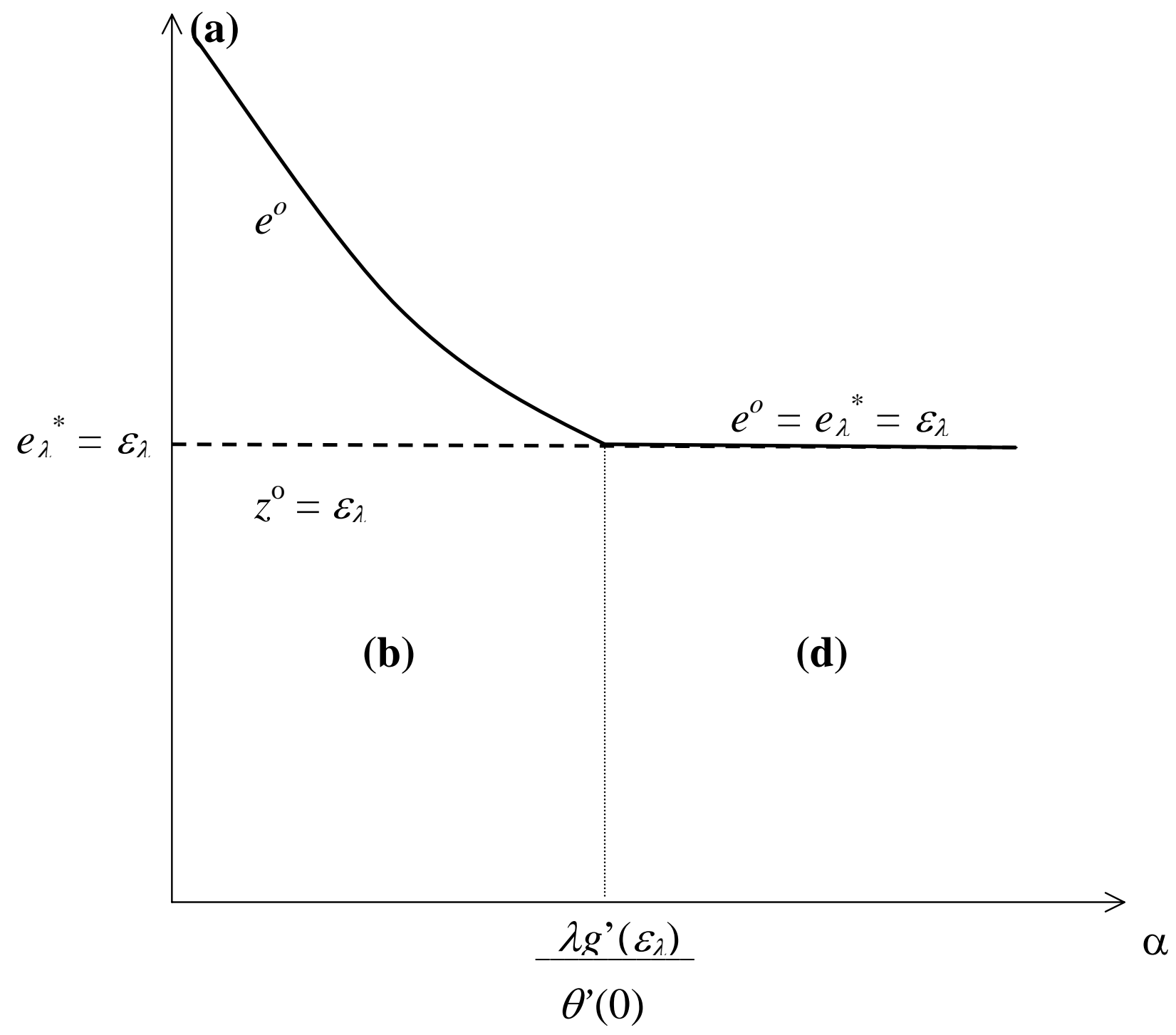

Figure 2: Firm's decisions for $e_{\lambda}{ }^{*}=\varepsilon_{\lambda}$ 


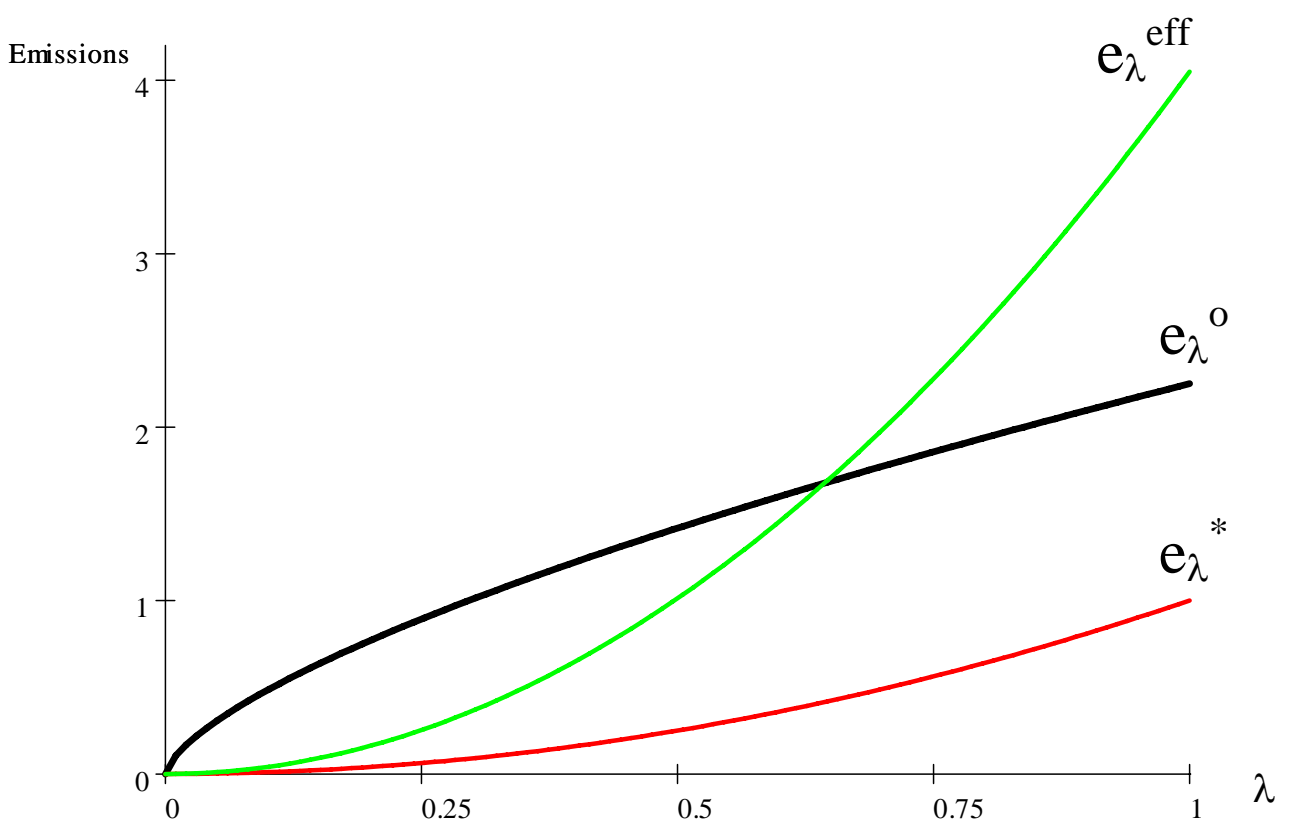

Figure 3: Comparison of the emission distributions for a low budget 


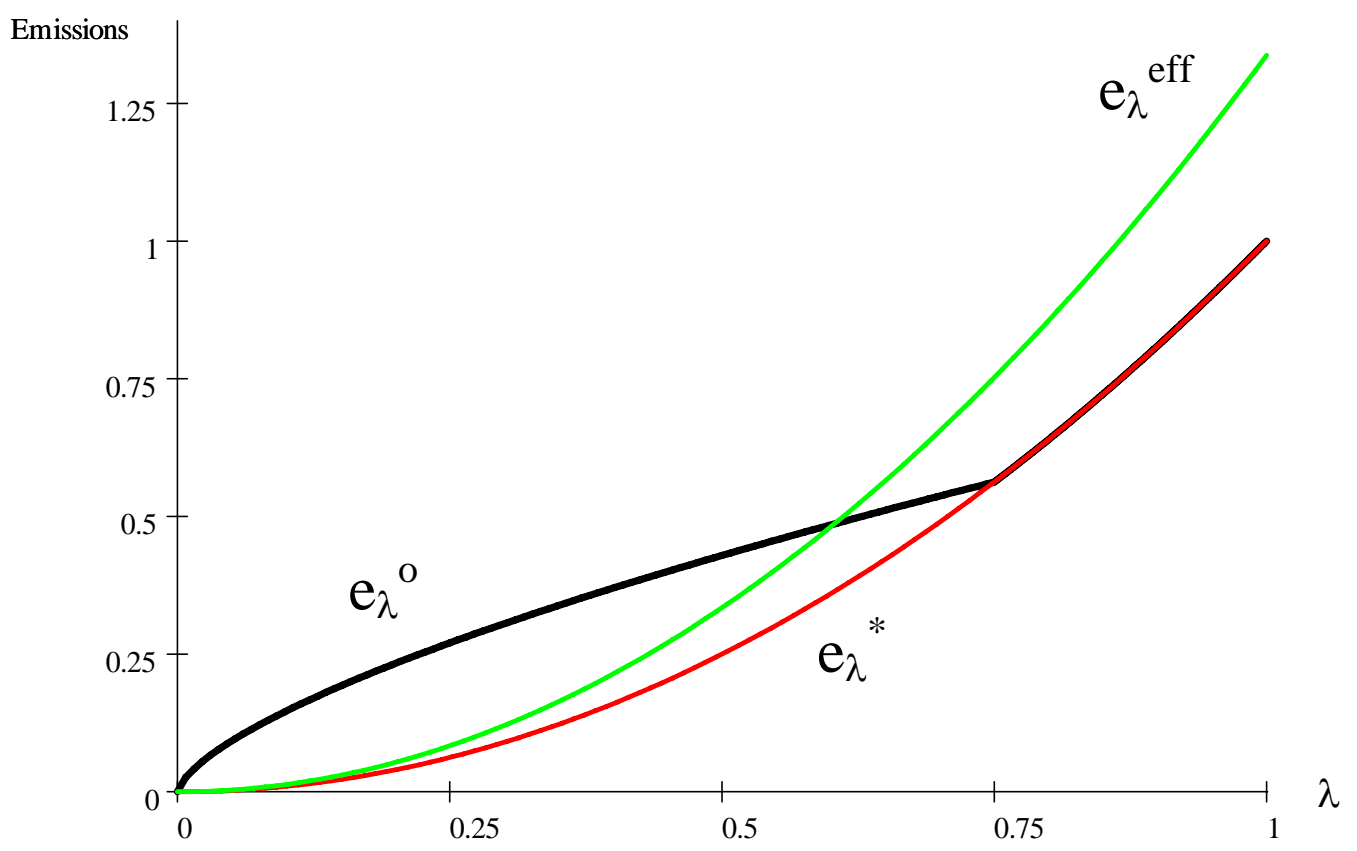

Figure 4: Comparison of the emission distributions for a large budget 